\title{
A conserved developmental program for sensory organ formation in Drosophila melanogaster
}

\author{
Nao Niwa ${ }^{1,4}$, Yasushi Hiromi ${ }^{1-3}$ \& Masataka Okabe $e^{1,2,4}$
}

Different sensory organs, such the eye and ear, are widely thought to have separate origins, guided by distinct organspecific factors that direct all aspects of their development. Previous studies of the $D$. melanogaster gene eyeless (ey) and its vertebrate homolog Pax6 suggested that this gene acts in such a manner and specifically drives eye development ${ }^{1,2}$. But diverse sensory organs might instead arise by segment-specific modification of a developmental program that is involved more generally in sensory organ formation. In D. melanogaster, a common proneural gene called atonal (ato) functions in the initial process of development of a number of segment-specific organs, including the compound eye, the auditory organ and the stretch receptor ${ }^{3,4}$, suggesting that these organs share an evolutionary origin. Here we show that $D$. melanogaster

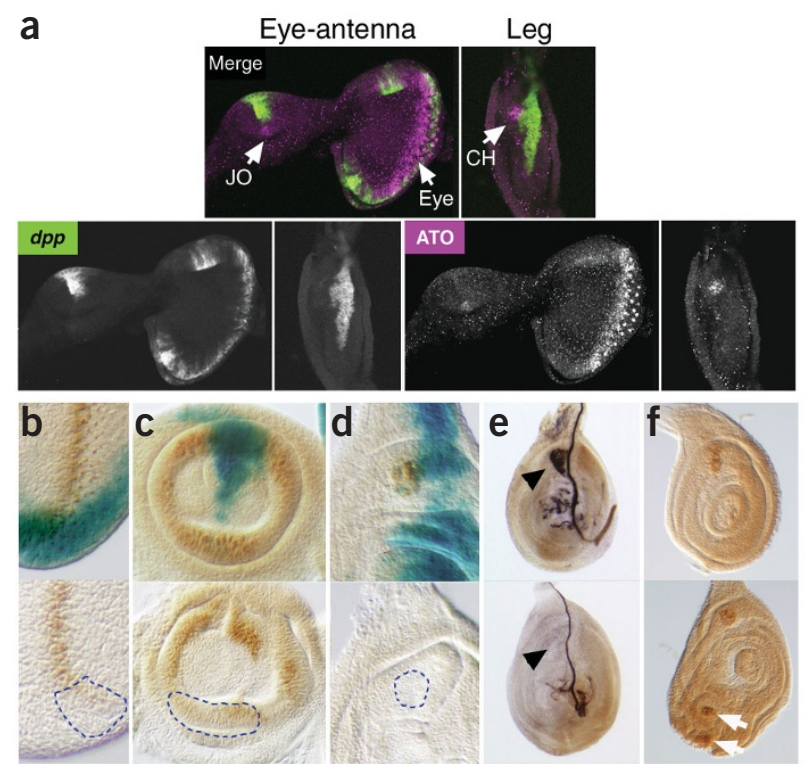

segment-specific sensory organs form through the integration of decapentaplegic (dpp), wingless (wg) and ecdysone signals into a single cis-regulatory element of ato. The induction of ectopic eyes by ey also depends on these signals for ato expression, and the ey mutant eye imaginal disc allows ato expression if cell death is blocked. These results imply that ey does not induce the entire eye morphogenetic program but rather modifies atodependent neuronal development. Our findings strongly suggest that various sensory organs evolved from an atodependent protosensory organ through segment specification by ey and Hox genes.

If multiple segment-specific sensory organs have a common origin, they may share an intrasegmental environment for their formation. We therefore examined whether there are any similarities in the conditions necessary for the formation of three ato-dependent sensory organs in D. melanogaster: the compound eye, Johnston's organ (auditory organ) in the antenna and the chordotonal organ (stretch receptor) in the leg. Spatiotemporal conditions for sensory organ development have been studied most extensively in the D. melanogaster compound eye.

Figure 1 The compound eye, auditory organ and stretch receptor share common spatiotemporal conditions for their formation. (a) Eye-antennal and leg imaginal discs of $d p p$-lacZ larvae $\sim 80 \mathrm{~h} \mathrm{AEL}$ stained with antibodies to ATO (magenta) and to $\beta$-galactosidase (green). The formation of eye in the eye imaginal disc, Johnston's organ ( $\mathrm{JO}$ ) in the antennal disc and the chordotonal organ $(\mathrm{CH})$ in the leg disc all start $80 \mathrm{~h} \mathrm{AEL}$ adjacent to the $d p p$ expression domain. (b-e) Imaginal discs of UAS-lacZ/dpp-Gal4 (top) and UAS-Dad/dpp-Gal4 (bottom) larvae. Blue region in the top panels of b-d show the areas where GAL4 drives Dad expression. Blocking the DPP signal caused loss of ATO expression (bottom panels) in the developing eye (b), Johnston's organ (c) and chordotonal organ (d). Use of a neuron-specific $22 \mathrm{C} 10$ antibody also showed that the expression of Dad blocked neural differentiation in the chordotonal organ (arrowhead) of the leg disc (e). (f) ATO expression in leg discs of wild-type (top) and UAS-pan $\Delta N / d p p$-Gal4 larvae (bottom). Blocking the WG signal caused ectopic ATO expression in the ventral region of the disc (arrows).

\footnotetext{
${ }^{1}$ Division of Developmental Genetics, National Institute of Genetics, 1111 Yata, Mishima, Shizuoka 411-8540, Japan. 2Department of Genetics, SOKENDAI, 1111 Yata, Mishima, Shizuoka 411-8540, Japan. ${ }^{3}$ CREST, Japan Science and Technology Agency, Japan. ${ }^{4}$ Present addresses: Morphogenetic Signaling group, RIKEN Center for Developmental Biology, 2-2-3 Minatojima-minamimachi, Chuo-ku, Kobe, Hyogo 650-0047, Japan (N.N.); MRC Centre for Developmental Neurobiology, King's College London, New Hunt's House, 4th Floor, Guy's Hospital Campus, London SE1 1UL, UK (M.O.). Correspondence should be addressed to M.O. (maokabe@lab.nig.ac.jp) or Y.H. (yhiromi@lab.nig.ac.jp).
} 

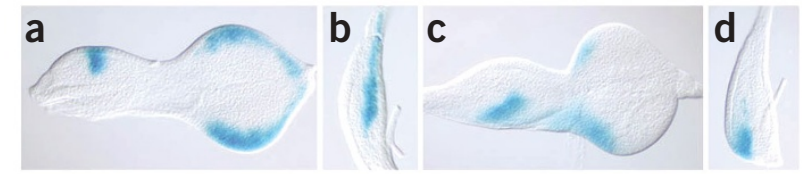

e
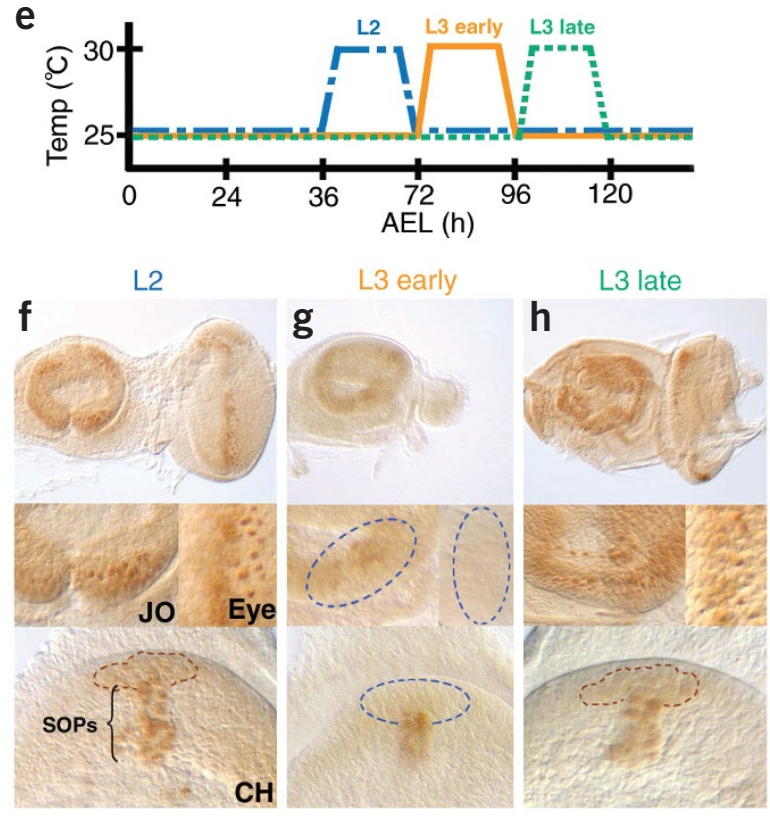

Compound eyes start to form at an early third instar larval stage at the posterior edge of the eye imaginal disc (Fig. 1a), where two morphogens, DPP and WG, determine the site of eye initiation; $d p p$ expressed at the posterior edge initiates eye development, and $w g$ at lateral edges inhibits eye development laterally ${ }^{5}$. We examined whether
Figure 2 The ecdysone signal provides the common temporal information for the formation of the eye, Johnston's organ and chordotonal organ. (a-d) Both $d p p$ and $w g$ are expressed before the initiation of sensory organ formation. Eyeantennal $(\mathbf{a}, \mathbf{c})$ and leg $(\mathbf{b}, \mathbf{d})$ discs $72 \mathrm{~h} \mathrm{AEL}$ in flies carrying $\operatorname{dpp}-/ a c Z(\mathbf{a}, \mathbf{b})$ or $w g-l a c Z(\mathbf{c}, \mathbf{d})$ reporter genes. (e-h) Ecdysone signal at $80 \mathrm{~h} \mathrm{AEL}$ is required for the formation of all sensory organs. (e) Temperature-shift scheme. (f-h) ATO expression in late third instar (L3 late) larvae. Top row, low-magnification view of the eye-antennal disc; middle and bottom rows, high-magnification of Johnston's organ (JO), the eye and the chordotonal organ $(\mathrm{CH})$ in the leg disc. The region circled in brown $(\mathbf{f}, \mathbf{h})$ indicates ATO expression in the proneural cluster of the chordotonal organ. Staining below it are sense organ precursors (SOPs) derived from the proneural cluster. ATO expression in the eye, Johnston's organ and chordotonal organ was lost only when the temperature was shifted at the early third stage (L3 early, $\mathbf{g}$ ). Circled in blue (g) is the region in which ATO-positive proneural clusters should have formed. The number of sense organ precursors was also decreased. The morphogenetic furrow did not form in $\mathbf{g}$; hence, the eye did not differentiate.

these conditions for eye formation also apply to Johnston's organ and the chordotonal organ. The forming Johnston's organ and chordotonal organ were detected as ATO-positive cell clusters $\sim 80 \mathrm{~h}$ after egg laying (AEL; Fig. 1a). These clusters were located adjacent to the $d p p$-expressing cells in regions where $w g$ was not expressed. When DPP signaling was blocked by the overexpression of Daughters against dpp (DAD), an intracellular negative regulator of DPP signaling ${ }^{6}$, ATO expression was lost from all imaginal discs, and neither Johnston's organ nor the chordotonal organ formed (Fig. 1b-e). Conversely, overexpression of a dominant-negative form of Pangolin (PAN, also called dTCF), a key transcription factor mediating the WG signal ${ }^{7}$, caused ectopic ATO expression in the ventral side of leg imaginal discs (Fig. 1f). These results indicate that the conditions that control eye formation also restrict the positions of Johnston's organ and the chordotonal organ.

Both Johnston's organ and the chordotonal organ formed simultaneously with the initiation of eye formation (Fig. 1a), suggesting that the induction of these three organs requires a temporal condition.
Figure 3 Common spatiotemporal conditions for the formation of multiple sensory organs act through a single $3^{\prime}$ enhancer of ato ( $\left.3^{\prime} E: 6.4\right)$. Imaginal discs of the transgenic flies carrying $3^{\prime} E: 6.4-l a c Z$ reporter genes were stained with an antibody to $\beta$-galactosidase. (a-d) DPP signal acts through two MAD binding sites (MAD BS) in the ato enhancer. Panels show, from left to right, eyeantennal disc (Ey-an), high magnification of Johnston's organ (JO), leg disc and high magnification of the chordotonal organ $(\mathrm{CH})$. (a) The $3^{\prime} E$ :6.4-lacZ expression pattern was identical to those of endogenous ato in the eye, Johnston's organ (arrows) and chordotonal organ. (b) Inactivation of either of the two MAD binding sites caused a marked reduction of lac $Z$ expression in the eye, Johnston's organ and chordotonal organ. (c) Mutating both MAD binding sites abolished enhancer activity. (d-g) The $3^{\prime} \mathrm{E}: 6.4$ genomic fragment receives the spatiotemporal conditions to induce ato expression during sensory organogenesis. Blocking the DPP signal caused loss of lac $Z$ expression in imaginal discs of UAS-Dad, 3'E:6.4-lacZ/dpp-GAL4 larvae (d). Blocking the WG signal resulted in ectopic lacZ expression in the leg disc of UASpan $\Delta N, 3^{\prime} E: 6.4-l a c Z / d p p-G A L 4$ larvae (e). The lacZ expression in both the eye (f) and the chordotonal organ (g) started $80 \mathrm{~h}$ AEL. a

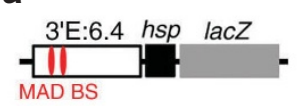

b

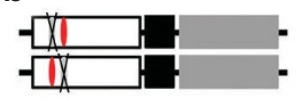

C

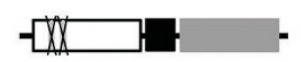

d
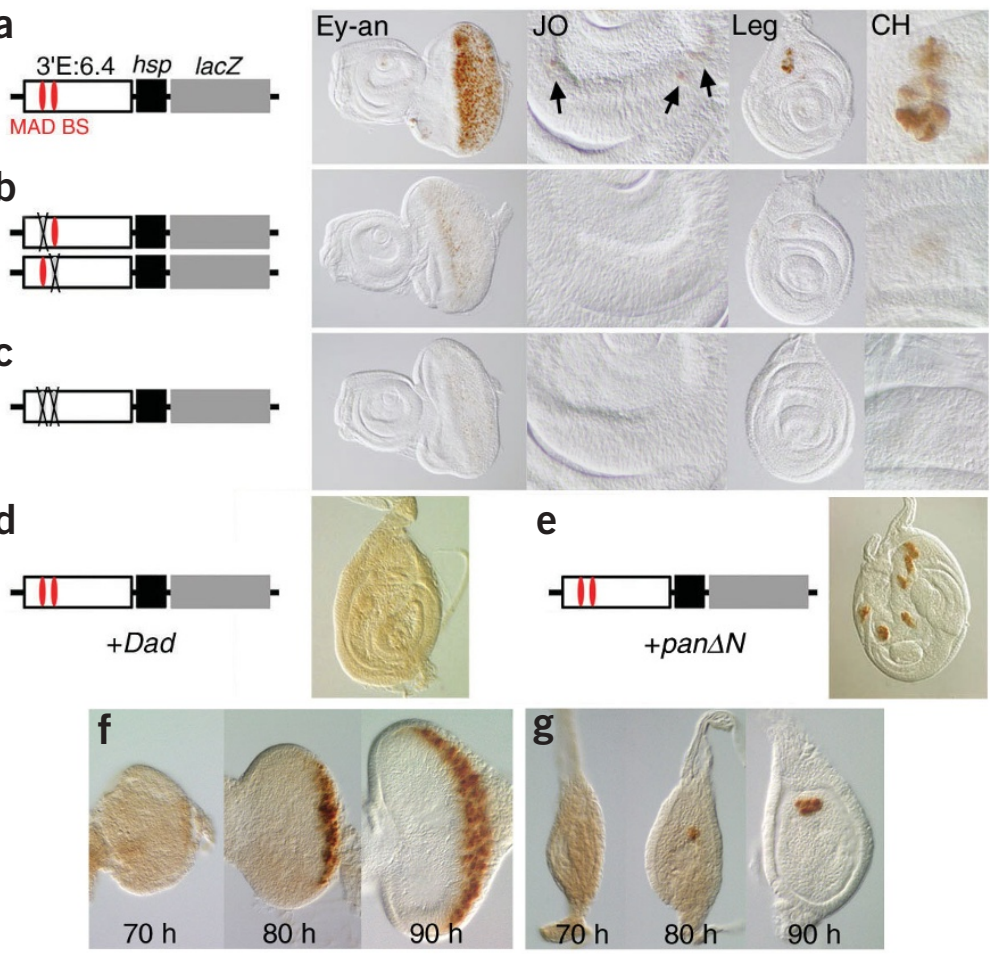
Because $d p p$ and $w g$ are expressed earlier than $80 \mathrm{~h}$ AEL and thus probably do not provide temporal specificity (Fig. 2a-d), we considered the hormonal factor ecdysone, as it is required for multiple aspects of eye morphogenesis in insects ${ }^{8}$. We used a temperature-sensitive allele of the ecdysoneless $(e c d)$ mutant, which affects ecdysone synthesis or release in the ring gland ${ }^{9}$, to reduce the ecdysone titer at various developmental stages (Fig. 2e). When the temperature was shifted at an early third instar larval stage (72-96 h AEL), ATO expression never initiated in each imaginal disc, and the eye, Johnston's organ and the chordotonal organ did not form (Fig. 2g). Exposure to the restrictive temperature at other stages had no effect on the formation of these sensory organs, although a temperature shift after the initiation of eye development affected the progression of the morphogenetic furrow as previously described ${ }^{10}$ (Fig. 2f,h). These results suggest that the temporal signal to initiate the formation of the eye, Johnston's organ and the chordotonal organ is provided by ecdysone.

A probable mechanism for the shared control of sensory organ development is for the common spatiotemporal information to be interpreted by a single gene. Because ato expression is controlled by these conditions, we analyzed its transcriptional regulatory sequences. Whereas the $5^{\prime}$ region of ato contains multiple autoregulatory enhancers that are specific to a particular sensory organ, the $3^{\prime}$ region is required for the initiation of expression in the compound eye, Johnston's organ and the chordotonal organ ${ }^{11}$ and is thus a good candidate for the common entry site for the spatiotemporal signals. A lac $Z$ reporter gene carrying a $6.4-\mathrm{kb}$ genomic fragment of the ato $3^{\prime}$ enhancer (3'E:6.4) initiated expression $80 \mathrm{~h}$ AEL in these organs in a pattern indistinguishable from that of ato itself (Fig. 3a,f,g). Furthermore, this enhancer responded to manipulations in DPP and
WG signals in the same way as endogenous ato (Fig. 3d,e). Thus, this 3'E:6.4 genomic fragment of ato integrates all known spatial and temporal signals involved in the control of shared aspects of sensory organ formation.

Within the 3'E:6.4 enhancer are two sites that match the consensus binding sequence for $\mathrm{MAD}$, a downstream transcription factor of the DPP signal ${ }^{12}$. Mutating either of the two sites substantially reduced lac $Z$ expression in all three sensory organs, and mutating both sites abolished 3'E:6.4 activity (Fig. 3b,c). These findings show that the two MAD binding sites in the ato regulatory element allow all three sensory organs to receive the DPP signal, implying that the target site of the DPP signal in D. melanogaster has been conserved during the diversification of sensory organs.

The conservation of a molecular basis for sensory organ formation may imply that the compound eye, Johnston's organ and the chordotonal organ all evolved from an ancestral ato-dependent sensory organ (protosensory organ). Derivation of diverse sensory organs from a protosensory organ is consistent with the idea that the body of an arthropod ancestor consisted of repeats of homogeneous segments ${ }^{13}$. In this putative worm-like ancestor, each segment must have possessed a protosensory organ that formed within the molecular environment common to all the segments. We expect, at least in arthropod evolution, that such a protosensory organ consisted of several scolopidia, neuronal units that constitute scolopophorous organs such as Johnston's organ and the chordotonal organ, because this structure is distributed widely among arthropod groups, and in adult insects, the scolopophorous sensory organs can be generated in every body segment ${ }^{13,14}$.

The formation of the eye in various organisms depends on PAX tran-
Figure 4 Ectopic eye formation by ey is restricted by conditions that determine sensory organ formation. (a,b) The frequency of ectopic eyes produced by ey expression at various stages during larval development is shown, scored by the expression of GBS-lacZ. The number of larvae examined for each treatment are indicated below. (a) The existence of at least one disc with ectopic GBS-lacZ expression was scored as a positive response. When various discs were scored separately (b), all discs examined had the same temporal response, with the strongest effect $\sim 80 \mathrm{~h}$ AEL (early third instar). (c-f) Spatial restriction in the response to ey. Imaginal discs in which ey was ubiquitously expressed using $h s-$ GAL4, by applying $1 \mathrm{~h}$ of heat-shock at $37^{\circ} \mathrm{C}$ during 72-96h AEL, are shown. All panels, except for $\mathbf{c}$, show a leg disc (top) and a wing disc (bottom) at late third instar larval stage. (c) Nonheat-shocked control discs. Ey-an, eye-antennal disc. Eye differentiation was visualized using a GBS-lacZ reporter (c,d) or the neuronal marker ELAV (e,f, arrowheads). Eye differentiation occurred only in several restricted regions in the leg and wing discs, whose positions within the discs were invariant despite subtle changes in the timing of heat shock (number of discs examined: leg discs, 3,400; wing discs, 1,100). Similar results were obtained with the earlier differentiation marker Dachshund (data not shown). Ectopic eyes were located adjacent to $d p p$-expressing cells (e) and far from the region that expressed $w g(\mathbf{f})$, both visualized using lac $Z$ reporter genes. (g) Similar spatial restrictions applied when ey was expressed in randomly generated clones. Although ey-expressing clones formed in various regions of the disc (white arrows, middle panel), the position that expressed neuronal marker ELAV (brown, left panel) was restricted. Right panel shows positions of all clones (solid circles) generated in leg and wing discs (13 discs each). Clones that included an ectopic eye are shown in red. (h,i) $d p p$ and wg restrict the site of ectopic eye induction. Coexpression of ey and a constitutively active form of DPP receptor Thickveins induced large ectopic eyes in regions far from the dpp expression domain (h). Coexpression of wg with ey completely blocked ectopic eye formation by ey (i). Expression was driven by dpp-GAL4(4A.3), in which GAL4 is broadly expressed in the posterior region of the leg and wing discs. ELAV+ cells in the leg disc (i) are precursors of endogenous bristles.$$
\text { a }
$$

e
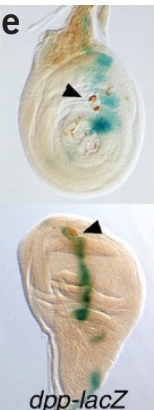
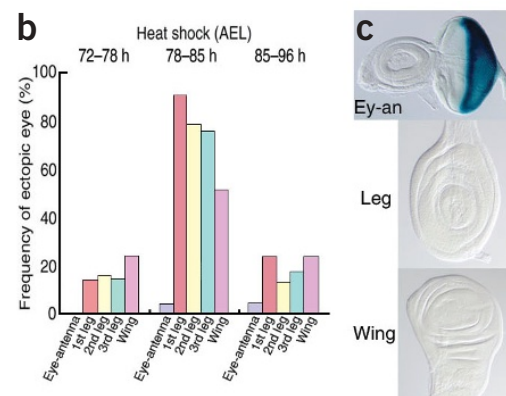

h

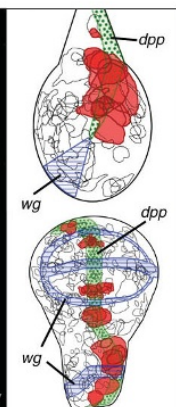

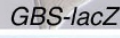
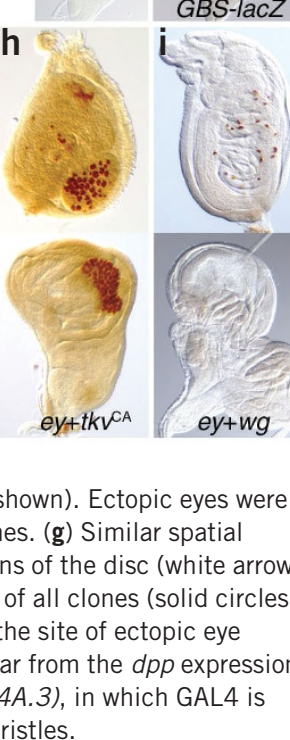
Figure 5 ey functions as a segmental identity gene for the eye segment rather than an eye-specific master control gene. (a-d) ATO expression in the eye disc can be uncoupled from eye differentiation in an ey mutant. $(\mathbf{a}, \mathbf{b})$ Acridine orange staining of wild-type (a) and ey ${ }^{2}$ mutant (b) early third instar larval eye disc showed severe apoptosis in the ey ${ }^{2}$ mutant disc. (c,d) Eye-antennal discs in which clones of cells coexpressing p35 and GFP were generated in $y$ w hsFLP; AyGAL4 UAS-P35 UASGFP; ey larvae. (c) No endogenous eye formed in this disc. (d) A partial eye was present in the ventral region. When apoptosis in the ey eye disc was blocked by overexpression of a caspase inhibitor p35 (green), groups of cells (arrows) were found that expressed ATO (red) but not eyespecific Glass (blue). Because these clusters do not form the morphogenetic furrow, which is an eye-specific feature, the cluster remained small, unlike the endogenous ATO-positive cluster, which spreads by furrow progression. We were unable to follow the differentiation of these ATO-positive cells, presumably because these cells are in a

'ground state' that does not correspond to any sensory organ present in $D$. melanogaster. (e,f) Ectopic ey expression produces not only the eye but also surrounding head structures. High magnification of the ectopic eyes formed in the wing blade (e) and in the leg tibial segment (f) of $y$ w hsFLP; AyGAL4/UAS-ey adults. Ectopic eyes are surrounded by long sensory bristles (black arrows). Leg bristles (white arrows) have specific structure called bracts, but bristles formed by ectopic ey are not associated with bracts and instead resemble hairs surrounding the endogenous eye. Red pigments in the ectopic eye (arrowhead in $\mathbf{e}$ ) leached out in the mounting medium. (g,h) A model for diversification of segment-specific sensory organs. In an ancestral arthropod, spatiotemporal signals such as DPP and WG directed the formation of an ato-dependent protosensory organ (g). The protosensory organ was subsequently modified by segmental identity genes such as ey and Hox genes, generating various segment-specific sensory organs (h). Whether the ecdysone signal induces ato expression directly or indirectly is unknown. JO, Johnston's organ; $\mathrm{CH}$, chordotonal organ.

scription factors Eyeless and PAX6 (ref. 2). The ability of ey to form eyes at ectopic positions led to the proposal that $e y$ is a 'master control gene' of the eye, controlling all aspects of eye formation ${ }^{2}$. If, as our results suggest, the eye, Johnston's organ and the chordotonal organ have a common origin, eye formation should depend not only on ey expression but also on the spatial and temporal conditions that controlled the formation of the protosensory organ ${ }^{15}$. To examine whether the ability of $e y$ to promote eye differentiation is restricted spatially or temporally, we expressed ey ubiquitously at various developmental stages. In all discs examined, ey was able to generate ectopic eyes only when expressed $\sim 80 \mathrm{~h}$ AEL, the time of endogenous sensory organ formation (Fig. 4a,b). This responsiveness to ey was also restricted spatially; ectopic eyes formed only in small groups of cells located close to the anterior-posterior compartment boundary where $d p p$ is expressed ${ }^{16}$ and distant from the expression domain of $w g$. This pattern is identical to the spatial relationships of endogenous eye and scolopophorous sensory organs with respect to these two morphogens (Fig. 4d-g). We also observed spatial restriction of ectopic eye formation when $e y$ was expressed randomly in clonal patches, indicating that this restriction is independent of the method of ey misexpression (Fig. 4g). To test whether DPP and WG regulate ectopic eye formation, we expressed ey under conditions where DPP or WG signals are artificially activated. Coexpression of ey and a constitutively active form of the DPP receptor Thickveins ${ }^{17}$ induced ectopic eyes in regions where $d p p$ is not expressed, and coexpression of $w g$ with ey completely blocked ectopic eye formation (Fig. $4 \mathbf{h}, \mathbf{i})$. Thus, the ability of $e y$ to generate an eye is not ubiquitous but is restricted by the same spatiotemporal conditions that are required for the formation of the endogenous eye and scolopophorous organs.

If the activity of Eyeless depends on the conditions that determine the formation of sensory organs in general, ey probably does not control all aspects of eye organogenesis. To address the function of $e y$ in eye formation, we reexamined the loss-of-function phenotype of the ey mutant, eyeless $^{2}\left(e y^{2}\right)$, which is an amorphic allele in the eye field ${ }^{1}$. Removing $e y$ activity in the eye imaginal disc caused extensive apoptosis $(\text { Fig. 5a,b })^{18}$, but if apoptosis was blocked, a cluster of ATO-expressing cells formed in $e y^{2}$ eye imaginal discs (Fig. 5c,d). Thus, ATO expression can be uncoupled from eye differentiation in an ey mutant eye disc, implying that these two processes are linked by $e y$. We propose that $e y$ modifies the preexisting genetic program of sensory organ formation to generate a specific sensory organ, the eye. Our results further suggest that ey functions parallel to or downstream of ATO expression and sensory organ formation, rather than being an upstream master control gene.

Modification of a protosensory organ probably occurred in an arthropod ancestor, accompanying morphological changes of homogeneous segments into diverse ones, a process governed by Hox or homeotic genes. The development of the posterior part of the head region depends on the activity of homeotic genes of the Antennapedia complex, but no homeotic gene has yet been identified for the eye segment, which includes both the compound eye and the surrounding head capsule ${ }^{19} . e y$ is a good candidate to have a Hox-like function in specifying the eye segment, because both the presumptive eye and the region that surrounds the eye express and require $e y^{20,21}$. Furthermore, ectopic expression of ey could produce head structures other than the eye, such as bristles (Fig. 5e,f). These results suggest that ey has a Hox-like function, specifying the eye segment rather than the eye alone ${ }^{22,23}$. During evolution, the appearance of segment-specific Hox genes probably modified not only the character of each segment along the anteroposterior axis but also the structure and function of the sensory organ contained in the segment (Fig. 5g,h). As the development of vertebrate sensory organs has many molecular similarities to that of sensory organs in D. melanogaster ${ }^{24-28}$, similar mechanisms of sensory organ diversification might also have functioned in the evolution of the vertebrate body structure. 


\section{METHODS}

Genetics. We used the following fly strains: $d p p$-lacZ BS3.0, wg gn11 ( $w g$-lacZ), dpp-GAL4 (4A.3), UAS-Dad, UAS-pan $\Delta N, e c d^{1}, D f(3 L) R-G 7, h s-G A L 4, U A S-$ $e y, U A S-t k v^{\mathrm{CA}}, U A S-w g, e y^{2}$ and UAS-P35. For original references, see Flybase $^{29}$. We induced clones expressing ey in the genotype $y$ whs-FLP; AyGAL4 UAS-ey UAS-GFP. To rescue cell death in ey mutant disc, we made the larvae of genotype $y$ whsFL; AyGAL4 UAS-P35 UAS-GFP; ey $^{2}$.

Immunohistochemistry. We obtained the following antibodies from Developmental Studies Hybridoma Bank, which were developed under the auspices of the NIHD: antibodies to ELAV 9F8A9 and to Glass 9B2.1 (developed by G.M. Rubin, University of California, Berkeley), 22C10 (developed by S. Benzer, California Institute of Technology) and antibody to b-galactosidase 40-1a (developed by J. Sanes, Washington University). Polyclonal antibody to ATO was a gift from Y.N. $\operatorname{~Jan}^{3}$ (University of California, San Francisco). Secondary antibodies were from the Jackson laboratory. We detected cell death by acridine orange staining ${ }^{30}$. To reduce the ecdysone titer, we shifted $e c d^{1} / D f(3 L) R$-G7 larvae up to the nonpermissive temperature $\left(30^{\circ} \mathrm{C}\right)$ during the second instar $(36-72 \mathrm{~h} \mathrm{AEL}$, L2), early third instar (72-96 h AEL, L3 early) or late third instar (96-120 h AEL, L3 late) larval stages. We dissected imaginal discs from wandering larvae (late L3) and stained them with antibody to ATO. For $e y$ misexpression under heat-shock control, we heat-shocked UAS-ey, GBS (glass binding site)-lacZ/hs-GAL4 larvae for $1 \mathrm{~h}$ and dissected and fixed imaginal discs at the late third instar stage. Ectopic eye formation was judged by the expression of GBS-lacZ.

Transgenic flies. We excised an ato $3^{\prime}$ enhancer from the DS03128 D. melanogaster $\mathrm{P} 1$ clone as a 6.4-kb BamHI-Bam HI fragment and cloned it into the pCaSpeR hs 43 lac $Z$ vector. We used site-directed mutagenesis to change two Mad binding sites ( $5^{\prime}$-ccgagtGCCGGCGattcga- $3^{\prime}$, located at $+2,451$ bp downstream of the ato transcription start site, and $5^{\prime}$-ggcagtGCCGCCGacacag- $3^{\prime}$, located at $+3,221 \mathrm{bp}$ ) to $5^{\prime}$-ccgagtCTAGAGCattcga- $3^{\prime}$ and $5^{\prime}$-ggcagtCTGCAGT acacag- $3^{\prime}$, respectively. We also cloned mutated $6.4-\mathrm{kb}$ genomic fragments into pCaSpeR hs43 lacZ. We constructed GBS-lacZ by inserting a NLS-lacZ gene into the GMR vector ${ }^{30}$. It expresses nuclearly localized $\beta$-galactosidase under the control of the eye-specific Glass protein.

Figure preparation. We prepared figures according to recommendations in "Barrier-free presentation that is friendly to colorblind people" (M.Okabe \& K. Ito; see URL below).

URLs. Flybase is available at http://flybase.bio.indiana.edu/. Barrier-free figure guidelines are available at http://jfly.iam.u-tokyo.ac.jp/color.

\section{ACKNOWLEDGMENTS}

We thank Y. N. Jan, W. Gehring, S. Goto, M. Nakamura, T. Tabata, the Developmental Studies Hybridoma Bank and the Bloomington Stock Center for antibodies and flies; all members of the Hiromi, Hotta and Hayashi laboratories at National Institute of Genetics for discussions; S. West for injecting the GBS-lacZ construct; and A. Graham and D. Sipp for comments on earlier versions of the manuscript. This work was supported by grants from the Ministry of Education, Science, Sports and Culture of Japan (Y.H. and M.O.) and Japan Society for the Promotion of Science (Research for the Future Program (Y.H.)).

\section{COMPETING INTERESTS STATEMENT}

The authors declare that they have no competing financial interests.

Received 13 November 2003; accepted 23 January 2004

Published online at http://www.nature.com/naturegenetics/
1. Halder, G., Callaerts, P. \& Gehring, W.J. Induction of ectopic eyes by targeted expression of the eyeless gene in Drosophila. Science 267, 1788-1792 (1995).

2. Gehring, W.J. \& Ikeo, K. Pax 6: mastering eye morphogenesis and eye evolution. Trends Genet. 15, 371-377 (1999).

3. Jarman, A.P., Sun, Y., Jan, L.Y. \& Jan, Y.N. Role of the proneural gene, atonal, in formation of Drosophila chordotonal organs and photoreceptors. Development 121 2019-2030 (1995).

4. Caldwell, J.C. \& Eberl, D.F. Towards a molecular understanding of Drosophila hearing. J. Neurobiol. 53, 172-189 (2002).

5. Hazelett, D.J., Bourouis, M., Walldorf, U. \& Treisman, J.E. decapentaplegic and wing less are regulated by eyes absent and eyegone and interact to direct the pattern of retinal differentiation in the eye disc. Development 125, 3741-3751 (1998).

6. Tsuneizumi, K. et al. Daughters against dpp modulates $d p p$ organizing activity in Drosophila wing development. Nature 389, 627-631 (1997).

7. van de Wetering, M. et al. Armadillo coactivates transcription driven by the product of the Drosophila segment polarity gene dTCF. Cell 88, 789-799 (1997).

8. Champlin, D.T. \& Truman, J.W. Ecdysteroids govern two phases of eye development during metamorphosis of the moth, Manduca sexta. Development 125, 2009-2018 (1998)

9. Henrich, V.C., Tucker, R.L., Maroni, G. \& Gilbert, L.I. The ecdysoneless (ecd $1^{\text {ts }}$ ) mutation disrupts ecdysteroid synthesis autonomously in the ring gland of Drosophila melanogaster. Dev. Biol. 120, 50-55 (1987).

10. Brennan, C.A., Ashburner, M. \& Moses, K. Ecdysone pathway is required for furrow progression in the developing Drosophila eye. Development 125, 2653-2664 (1998).

11. Sun, Y., Jan, L.Y. \& Jan, Y.N. Transcriptional regulation of atonal during development of the Drosophila peripheral nervous system. Development 125, 3731-3740 (1998).

12. Kim, J., Johnson, K., Chen, H.J., Carroll, S. \& Laughon, A. Drosophila Mad binds to DNA and directly mediates activation of vestigial by Decapentaplegic. Nature $\mathbf{3 8 8}$ 304-308 (1997).

13. Snodgrass, R.E. Principles of Insect Morphology (McGraw-Hill Book Company, New York, 1935).

14. Hallberg, E. \& Hansson, B.S. Arthropod sensilla: morphology and phylogenetic considerations. Microsc. Res. Tech. 47, 428-439 (1999).

15. Chow, R.L., Altmann, C.R., Lang, R.A. \& Hemmati-Brivanlou, A. Pax6 induces ectopic eyes in a vertebrate. Development 126, 4213-3222 (1999).

16. Chen, R., Halder, G., Zhang, Z. \& Mardon, G. Signaling by the TGF- $\beta$ homolog decapentaplegic functions reiteratively within the network of genes controlling retinal cell fate determination in Drosophila. Development 126, 935-943 (1999).

17. Adachi-Yamada, T. et al. p38 mitogen-activated protein kinase can be involved in transforming growth factor beta superfamily signal transduction in Drosophila wing morphogenesis. Mol. Cell. Biol. 19, 2322-2329 (1999).

18. Quiring, R., Walldorf, U., Kloter, U. \& Gehring, W.J. Homology of the eyeless gene of Drosophila to the Small eye gene in mice and Aniridia in humans. Science 265 785-789 (1994).

19. Rogers, B.T. \& Kaufman, T.C. Structure of the insect head in ontogeny and phylogeny: A view from Drosophila. Int. Rev. Cytol. 174, 1-84 (1997)

20. Halder, G. et al. Eyeless initiates the expression of both sine oculis and eyes absent during Drosophila compound eye development. Development 125, 2181-2191 (1998)

21. Kronhamn, J. et al. Headless flies produced by mutations in the paralogous Pax6 genes eyeless and twin of eyeless. Development 129, 1015-1026 (2002).

22. Matsuo, T. et al. A mutation in the Pax- 6 gene in rat small eye is associated with impaired migration of midbrain crest cells. Nat. Genet. 3, 299-304 (1993).

23. Grindley, J.C., Davidson, D.R. \& Hill, R.E. The role of Pax-6 in eye and nasal development. Development 121, 1433-1442 (1995).

24. Bermingham, N.A. et al. Math1: an essential gene for the generation of inner ear hair cells. Science 284, 1837-1841 (1999).

25. Brown, N.L., Patel, S., Brzezinski, J. \& Glaser, T. Math5 is required for retinal ganglion cell and optic nerve formation. Development 128, 2497-2508 (2001).

26. Kay, J.N., Finger-Baier, K.C., Roeser, T., Staub, W. \& Baier, H. Retinal ganglion cell genesis requires lakritz, a zebrafish atonal homolog. Neuron 30, 725-736 (2001).

27. Furuta, Y. \& Hogan, B.L. BMP4 is essential for lens induction in the mouse embryo. Genes Dev. 12, 3764-3775 (1998).

28. Wawersik, S. et al. BMP7 acts in murine lens placode development. Dev. Biol. 207, 176-188 (1999).

29. FlyBase. The FlyBase database of the Drosophila genome projects and community literature. Nucleic Acids Res. 27, 85-88 (1999).

30. Hay, B.A., Wolff, T. \& Rubin, G.M. Expression of baculovirus P35 prevents cell death in Drosophila. Development 120, 2121-2129 (1994). 